\title{
Editorial
}

\section{Improving quality of life in patients with angina}

Angina appears to have a particularly deleterious effect on a patient's quality of life. In a community survey in which the short form 36 (SF36) was administered to 1200 patients with 11 different chronic illnesses (including arthritis, sciatica, back pain, diabetes, depression, and stroke) only patients suffering from chronic anxiety reported a more severe detrimental effect on their general health. ${ }^{1}$ These patients were already receiving medical treatment, what more could have been done to help them?

\section{Interventional treatments}

Both coronary artery bypass grafting and angioplasty provide worthwhile reductions in symptoms and reduce early mortality in selected patients. The relative merits of each procedure are likely to be debated for some time but, whatever the final consensus, without a huge increase in resources, neither intervention will help most angina patients. In the UK there are approximately 1.6 million people with angina, ${ }^{2}$ of whom approximately $20 \%$ are referred to secondary care $^{3}$ and $2 \%$ receive surgery or angioplasty each year. ${ }^{2}$ The long waiting lists for referral to secondary care, the scarcity of cardiologists, and an aging population make it likely that, for some time to come, the vast majority of patients with angina will remain unknown to secondary care and will not be considered for surgery or angioplasty.

\section{Care in the community}

Two recent community based studies have demonstrated that improved care can produce significant improvements in both clinical status and quality of life. In the first of these studies 688 primary care patients with angina were randomised to health education delivered by health visitors, or to routine medical care. The study produced improvements in the educational group in: activity levels, compliance with medication, lifestyle, and quality of life. ${ }^{4}$ In the second study, 1173 patients, of whom $50 \%$ had angina, were recruited from 19 general practices in Aberdeenshire. ${ }^{5}$ Practice nurses worked to a specially designed treatment manual to review medical treatment and negotiate behaviour change with patients. Intervention patients had significant improvement in blood pressure, lipids, and aspirin use, a $28 \%$ reduction in hospital admissions, and an improvement in quality of life. ${ }^{5}$ Both of these interventions were largely educational, neither included a formal exercise component or psychological interventions, two of the main components of cardiac rehabilitation. Meta-analyses and systematic review suggest that, in myocardial infarction patients, rehabilitation improves quality of life and reduces cardiac and all cause mortality by 20-25\%. ${ }^{6}$ Could cardiac rehabilitation provide similar gains for angina patients?

\section{Cardiac rehabilitation}

Trials of cardiac rehabilitation programmes specifically designed for angina patients have shown considerable promise. For example, an exercise trial that crossed over patients from $\beta$ blockade to exercise, showed the antianginal benefits of exercise to be equal to that of $\beta$ blockade. ${ }^{7}$ In the lifestyle trial, a rehabilitation programme in which the main elements were exercise, stress management, and a low fat diet, the intervention patients reported a $90 \%$ reduction in angina at one year and $82 \%$ showed some degree of regression of coronary artery disease. ${ }^{8}$ In a trial of a specially developed angina management programme conducted by our group, patients reported a $70 \%$ reduction in episodes of angina and a $72 \%$ improvement in quality of life at one year. Of those awaiting elective bypass surgery on entry to the programme $50 \%$ were subsequently removed from the waiting list after independent review by their cardiologist $^{9}$ and the vast majority of these patients have not required any further cardiological intervention 3-7 years after treatment.

The latter trials were distinguished by their addition of psychological treatments, such as stress management and relaxation therapy, to exercise. There have also been a number of purely psychological interventions for angina, these have been reviewed elsewhere. ${ }^{10}$ The two treatments that showed the most promise were relaxation therapy and stress management. ${ }^{10}$ It appears that rehabilitation might provide substantial gains for patients. Unfortunately, cardiac rehabilitation programmes in the UK are underprovided, underresourced, and poorly supported by medicine. ${ }^{11}$ They usually only enrol postmyocardial infarction or cardiac surgery patients referred from secondary care, and currently treat fewer than $50 \%$ of these patients. Angina and heart failure are often used to exclude patients from cardiac rehabilitation, this is ironic as they are two groups who particularly benefit from an increased exercise capacity. There is a clear rationale for exercise training but what benefits can psychological treatments provide?

\section{Psychological factors in the production of angina}

Psychological factors have been associated with angina since its description. Herberden named it after the choking sensation of fear that accompanied each attack. John Hunter described how an 18th century surgeon's everyday worries - for example, that his bees might have swarmed while he was up in town, could provoke angina, and modern Holter monitoring studies confirm that as many as $50 \%$ of ischaemic episodes may be triggered by emotion rather than exertion. ${ }^{12}$ It is possible that this is through an interaction between raised autonomic drive and vasoconstriction. A number of other dynamic factors such as decreased myocardial contractility, increased afterload and alkalosis due to emotionally induced hyperventilation may also be involved. ${ }^{10}$ In a number of studies, questionnaire measures of anxiety, depression, and neuroticism have all shown moderate, positive correlations with the frequency and severity of the self report of angina, independent of the extent of coronary artery disease, ${ }^{11}$ which in itself is not predictive of self reported angina or disability. The success of medical treatment is also linked to psychological factors; at Duke University Medical Centre, Williams et al were able to predict prospectively $85 \%$ of patients who would succeed on medical treatment and $61 \%$ of those who would not, using an empirically derived model, the main element of which was the score on a questionnaire measure of hypochondriasis. ${ }^{13}$ The authors noted that it is these patients, who are not different on any measurable cardiological feature, who constitute the bulk of those referred on from primary care to cardiology for further 
investigations due to insufficient symptom relief from medical treatment.

\section{What is so bad about angina?}

This may appear a somewhat redundant and insensitive question, the obvious answer being "the pain and discomfort it causes", but this does not accord with the data. In the community survey of quality of life, ${ }^{1}$ the angina patient's pain score was less than half that of patients with sciatica, arthritis, and back pain, and roughly similar to patients with chronic anxiety states. Yet, although objectively the prognosis for the average stroke patient is far worse than that for an angina patient, the angina patients scored their general health as being twice as poor as did patients with stroke ${ }^{1}$ and lower than all other patients except those with chronic anxiety. Angina appears to have a disproportionately severe impact on a patient's view of their health status. With the development of sophisticated imaging techniques "angor animi" may no longer be regarded as a strong diagnostic sign of angina pectoris, but there is no reason to believe that the acute anxiety and the fear of death that accompanies angina is any less real or has any less impact on quality of life, than it did in 1772 .

In the past few years it has become increasingly clear that such beliefs and attributions are important predictors of outcome. For example, in a study of 400 patients who failed to return to work following uncomplicated myocardial infarction, Wynn claimed that in $40 \%$ of cases the reason could be directly attributed to an immanent fear of death due to an overcautious prognosis. ${ }^{14}$ In a further $23 \%$ of cases the patients' anxiety was directly related to a cardiac misconception such as, "every attack of angina causes further damage to my heart". ${ }^{14}$ In several surveys it has been shown that $80 \%$ of patients believe that the primary cause of their heart disease was worry or stress or overwork. In the face of such beliefs the most obvious coping strategy is to rest and to avoid the demands, stresses, and excitements and thus often the rewards, of a normal life. ${ }^{15}$ Unfortunately, many health professionals share these misconceptions and reinforce them, urging patients to avoid provoking angina, to rest and to "take things easy". Most physicians are now aware that rest is an inappropriate prescription for back pain, and angina may be the only remaining medical condition for which rest is (often unintentionally) prescribed.

Our experience in developing and evaluating the angina management programme is that it is important to educate patients about the true causes of angina, to reduce their exaggerated health worries, and to give them hope that through lifestyle changes they can fight back against premature death. It is important to provide specific advice and guidance about the lifestyle changes required and to encourage them to return to as normal a life as possible. Most of our patients have had a number of years of living with angina, usually becoming increasingly disabled by it. It seems possible to many of them and reasonable to us, that if better advice and guidance were given to patients close to the time of diagnosis, fewer of them would lapse into the fearful and restricted lifestyle that leads to reduced quality of life. We are currently evaluating this possibility in a controlled trial of a very brief educational intervention suitable for use in primary or secondary care shortly after diagnosis.

\section{Conclusion}

There is much that could be done to improve quality of life for the average angina patient. Improved cardiac education and hospital based rehabilitation programmes have both demonstrated worthwhile benefits. To cope with the numbers involved new community based rehabilitation programmes should be developed for patients with chronic stable angina. It has been shown that self help rehabilitation programmes can be delivered by specially trained practice nurses or health visitors, working from manuals, ${ }^{5} 16$ and this may be a practical and cost effective approach. Patients with more complex problems or those who do not respond to simple community based rehabilitation methods should be referred to more intensive rehabilitation programmes in secondary care. These programmes require greater investment in staff training and resources. Patients' beliefs about heart disease are often the most important determinant of their disability and quality of life. These factors should be addressed by medical staff at the point of diagnosis and throughout the illness career. It is important to emphasise that death is not imminent, that many risk factors are controllable by the patient and doctor, with very worthwhile clinical benefits, and that there is no evidence that brief periods of ischaemia are damaging. The common misconceptions that lead to undue anxiety and to the abandoning of enjoyable activities - for example, that each episode is a mini-heart attack, or that the cure is to rest, should be sought out and changed in the patient and wherever possible their family. A programme of daily walking, starting within the patients' current ability and being stepped up within the patients' comfort zone as their fitness increases, is simple to organise and to monitor. Above all clinicians should try to imbue patients with a sense of optimism and control over their fate by encouraging an active participation in the management of the condition.

Although I take full responsibility for any inaccuracies or opinions I would like to thank Professor Brian Pentecost, Professor Keith Fox, and Dr Iain Todd for their helpful comments and suggestions on earlier drafts of this manuscript.

R J P LEWIN

British Heart Foundation Rehabilitation Research Unit,

Department of Health Studies, The University of York,

Genesis 6, York Science Park, Heslington, York YO10 5DG, UK

email.rjpl1@york.ac.uk

1 Lyons RA, Lo SV, Littlepage BNC. Comparative health status of patients with 11 common illnesses in Wales. F Epidemiol Community Health 1994;48:388-90.

2 British Heart Foundation Health Promotion Research Group. Coronary heart disease statistics. London: British Heart Foundation, 1998.

3 Ghandi MM, Lampe C, Wood DA. Incidence, clinical characteristics, and short-term prognosis of angina pectoris. Br Heart f 1995;73:193-8.

4 Cupples ME, McKnight A. Randomised controlled trial of health promotion in general practice for patients at high cardiovascular risk. $B M \mathcal{F}$ promotion in gene

5 Campbell NC, Thain J, Deans HG, et al. Secondary prevention clinics for coronary heart disease: randomised trial of effect on health. $B M F$ 1998;316:1434-7.

6 NHS Centre for Reviews and Dissemination. Effective health care-cardiac rehabilitation, volume 4, number 4. York: University of York, 1998.

7 Todd IC, Ballantyne D. Antianginal efficacy of exercise training: a comparison with $\beta$ blockade. Br Heart F 1990;64:14-19.

8 Ornish D, Brown SE, Scherwitz LW, et al. Can lifestyle changes reverse coronary heart disease? Lancet 1990;336:129-33.

9 Lewin B, Cay EL, Todd I, et al. The angina management programme: a rehabilitation treatment. $\mathrm{Br} \mathcal{7}$ Cardiol 1995;1:221-6.

10 Lewin B. The psychological and behavioural management of angina. $\mathcal{F}$ Psychosom Res 1997;5:452-62.

11 Lewin RJ, Ingleton R, Newens AJ, et al. Adherence to cardiac rehabilitaion guidelines: a survey of cardiac rehabilitation programmes in the United Kingdom. BMF 1998;316:1354-5.

12 Deanfield JE, Maseri A, Selwyn AP, et al. Myocardial ischaemia during daily life in patients with stable angina: its relation to symptoms and heart rate changes. Lancet 1983;ii:753-8.

13 Williams RB, Haney TL, McKinnis RA, et al. Psychosocial and physical predictors of anginal pain relief with medical management. Psychosom Med 1986;48:200-10

14 Wynn A. Unwarranted emotional distress in men with ischaemic heart disease. Med f Aust 1967;2:847-51.

15 Fielding R. Patients' beliefs regarding the causes of myocardial infarction: implications for information-giving and compliance. Patient Education and Counselling 1987;9:121-34.

16 Lewin B, Robertson IH, Cay EL, et al. Effects of a self-help post-myocardialinfarction rehabilitation on psychological adjustment and use of health services. Lancet 1992;339:1036-40. 